\title{
Nueva especie de escorpión del género Hadruroides (Scorpiones: Caraboctoninae) de los valles interandinos de Perú
}

\section{A new scorpion species of the genus Hadruroides (Scorpiones: Caraboctoninae) from inter Andean valleys of Peru}

\author{
José Antonio Ochoa ${ }^{1,2}$ y Juan Carlos Chaparro'
}

\begin{abstract}
1 Museo de Historia Natural, Universidad Nacional de San Antonio Abad del Cusco, Paraninfo Universitario, Plaza de Armas s/n, Universitario,

Cusco, Perú. jaochoac2000@yahoo.com

Email Juan C. Chaparro: jchaparroauza@yahoo.com

2 Dirección actual: Division of Invertebrate Zoology, American Museum of Natural History, Central Park West at 79th Street, New York, NY 10024-5192, USA.
\end{abstract}

Presentado: $\quad$ 19/10/2007 Aceptado: $\quad 05 / 12 / 2007$

Publicado online: $21 / 07 / 2008$

\begin{abstract}
Resumen
Se describe Hadruroides bustamantei, una nueva especie de Caraboctoninae de los valles interandinos del centro del Perú (2600-3289 m). Esta especie está muy relacionado con $H$. mauryi Francke \& Soleglad, con la cual fue confundida. La nueva especie difiere de $H$. mauryi, por la proporción largo /ancho de la pinza del pedipalpo y en el patrón de pigmentación de los tergitos, patas y segmentos caudales. Con la descripción de $H$. bustamentei, el número de especies conocidas en el género Hadruroides se eleva a diez.
\end{abstract}

Palabras clave: Scorpiones, Caraboctoninae, Hadruroides, Perú, Andes.

\section{Abstract}

Hadruroides bustamantei, a new caraboctonid species from inter Andean valleys of central Peru (2600-3289 $\mathrm{m}$ ) is described. This species is most related to $H$. mauryi Francke \& Soleglad, with which was confused. The new species differs from $\mathrm{H}$. mauryi, by length/width ratio of the male chela and the pigmentation pattern of the tergites, legs and metasomal segments. With the description of $H$. bustamantei, the number of known species in the genus Hadruroides is elevated to ten.

Keywords: Scorpiones, Caraboctoninae, Hadruroides, Peru, Andes.

\section{Introducción}

El género Hadruroides Pocock, 1893, es uno de los grupos de escorpiones más representativos y característicos del Perú y el sur de Ecuador, la mayoría de sus especies se distribuyen en las vertientes occidentales de los Andes y son de fácil hallazgo en alrededores de ciudades como Lima, Trujillo, Chiclayo y Piura, pero también están presentes en valles interandinos de los Andes. Hasta la fecha fueron reportadas nueve especies del género Hadruroides, distribuidas en Ecuador y Perú, incluyendo una especie endémica para las Islas Galápagos (Maury, 1975; Sissom y Fet, 2000). El género fue citado para Colombia y Bolivia (Mello-Leitão, 1945; Karsch, 1879), pero su presencia en estos países es considerada dudosa (Maury, 1975; Francke y Soleglad, 1980; Flórez, 1990; Acosta y Ochoa, 2002), pero es muy probable que este presente en el norte de Chile.

La sistemática del género siempre ha sido tratada como confusa, incluso muchos autores han considerado la existencia de una sola especie (Kraepelin, 1894, 1899; Mello Leitão, 1945; Aguilar y Meneses, 1970). Pero gracias al trabajo de Maury (1975), se llegó a aclarar de mejor manera la confusión taxonómica que rondo en este género por más de 100 años, ello se debió más que todo a que Maury, pudo disponer de los ejemplares tipo de varias especies antiguas, que habían pasado a sinonimia de $H$. lunatus, y terminó separando al género en seis entidades: H. lunatus (L. Koch, 1867); H. carinatus Pocock, 1900; H. charcasus (Karsch, 1879); H. leopardus Pocock, 1900; H. maculatus (Thorell, 1876) y H. maculatus galapagoensis Maury, 1975. Sin embargo algunas dudas no fueron aclaradas, tal es el caso de la posición incierta de $H$. parvulus (Karsch, 1879) y H. robustus (Boeris, 1889). Posteriormente fueron añadidas al elenco del género: $H$. aguilari Francke \& Soleglad, 1980; H. mauryi Francke \& Soleglad, 1980 (Francke y Soleglad, 1980) y finalmente H. udvardyi Lourenço, 1995 (Lourenço, 1995).

Si bien algunas especies como $H$. lunatus presentan una amplia distribución y son relativamente bien conocidas, las otras especies necesitan una revisión sistemática exhaustiva, muchas de las diagnosis específicas son confusas y aparentemente algunas entidades incluyen más de una especie. Muestreos en los Andes centrales del Perú en el departamento de Ayacucho, nos han permitido capturar algunos ejemplares de Hadruroides que inicialmente fueron confundidas como $H$. mauryi (descrita originalmente para los departamentos de Cusco, Ayacucho y Huancavelica), sin embargo la revisión de los ejemplares tipo de $H$. mauryi y material adicional de la zona de Ayacucho, nos permiten confirmar que los ejemplares provenientes de Ayacucho y Huancavelica (incluyendo todos los paratipos de $H$. mauryi, excepto un juvenil de la localidad de Paruro) corresponden a una forma innominada (que describimos en el presente trabajo) y $H$. mauryi estaría restringida solamente a los valles interandinos del Cusco.

El género Hadruroides actualmente está ubicado dentro de la subfamilia Caraboctoninae Kraepelin, 1905, que incluye además al género Caraboctonus Pocock, 1893, que tiene una distribución en la zona central de Chile. El rango nomenclatural de este grupo ha cambiado sustancialmente en los ultimos ańos. Hasta 1981 fue ubicada dentro de Vaejovidae, en dicho año Francke y Soleglad (1981) la incluyen dentro de la familia Iuridae, donde permaneció por un poco más de 20 años. Sin embargo recientemente Hadruroides y Caraboctonus junto al género norteamericano Hadrurus Thorell, 1876, fueron asignados en una familia separada (Soleglad y Fet, 2003), elevando a rango de familia a Caraboctoninae; no obstante Prendini y Weeler (2005) ubican nuevamente estos géneros en Iuridae, pero pocos meses después en el mismo año Fet y Soleglad (2005) restablecen los cambios hechos por Prendini y Weeler. Ello muestra sin duda una confusión taxonómica en el grupo y por tanto requiere un estudio más profundo, que involucre un análisis cladístico del género y refleje las relaciones de parentesco entre sus integrantes y su posición filogenética frente a otros grupos de escorpiones. Sin animo a discutir los últimos cambios sistemáticos a nivel de familia, preferimos temporalmente no asignar este grupo a ninguna de las familias propuestas.

\section{Métodos}

La terminología utilizada en este trabajo corresponde a Stahnke (1970) para morfología general, Vachon (1974) 
para nomenclatura de la tricobotriotaxia, y Vachon (1952) y Stahnke (1970), para nomenclatura de las carenas del caparax y pedipalpos.

\section{Abreviaturas}

Carenas en el caparax: $\mathrm{MA}=$ Medianas anteriores, $\mathrm{Mc}=$ Medianas centrales, LC= Laterales centrales. Carenas de los segmentos caudales I-V: DL= Dorsal lateral, LsM= Lateral supramediana, LM= Lateral mediana, LIM= Lateral inframediana, $\mathrm{V}_{\mathrm{L}}=$ Ventral lateral, VSM= Ventral submediana, $\mathrm{VM}_{\mathrm{M}}=$ Ventral mediana. Carenas de los pedipalpos: $\mathrm{DI}=$ Dorsal interior, $\mathrm{DE}=$ Dorsal exterior, $\mathrm{VI}=$ Ventral interior.

Colecciones: AMNH: American Museum of Natural History, New York, USA. CDA: Cátedra de Diversidad Animal I, Facultad de Ciencias Exactas, Físicas y Naturales, Universidad Nacional de Córdoba, Argentina. MHNC: Museo de Historia Natural, Facultad de Ciencias Biológicas, Universidad Nacional de San Antonio Abad del Cusco, Perú. MUSM: Museo de Historia Natural, Universidad Nacional Mayor de San Marcos, Lima, Perú.

\section{Hadruroides bustamantei $\mathrm{n} . \mathrm{sp}$.}

\section{(Figuras 1-17)}

Hadruroides mauryi: Francke \& Soleglad, 1980: 8 (part. error de determinación, ejemplares referidos a Huancavelica y Ayacucho); Sissom \& Fet, 2000: 413 (part.); Ochoa, 2003: 61 (part. error de determinación, ejemplares referidos a Ayacucho).

\section{Serie típica}

Holotipo macho (MHNC); 1 macho, 2 hembras, 1 juvenil paratipos (MHNC); 1 macho paratipo (MUSM); 1 macho, 1 hembra paratipos (CDA 000.158); Perú, Departamento Ayacucho, Provincia Huanta (cerca a la ciudad, 2630 m; 120 57' 18" S, 740 14'35" O), 20 diciembre 1998, J. Achicahuala Z. $\&$ J. A. Ochoa - 3 hembras, 4 juveniles paratipos (AMNH); Perú, Departamento Huancavelica, Provincia Churcampa, 15 $\mathrm{km}$ al norte de Anco (2700 m, río Mantaro), 25 julio 1971, O. F. Francke.

Diagnosis.- Morfológicamente está muy relacionada con H. mauryi (con la cual fue confundida). Se diferencia de esta especie por el patrón de pigmentación y la proporción largo / ancho de la pinza del pedipalpo. H. bustamantei n. sp. presenta dos manchas irregulares paramedianas y dos laterales sobre los tergitos I-VII, las cuales forman cuatro bandas longitudinales bien notorias; mientras que en $H$. mauryi, a pesar de tener una coloración más oscurecida, no presenta evidencia de un patron de pigmentación (diseño de manchas), a lo sumo algunas manchas ligeras en la margen posterior de los primeros tergitos. Adicionalmente $H$. bustamantei n. sp. presenta manchas prolaterales en todas las patas y el pigmento sobre las carenas de los segmentos caudales es bien evidente; por el contrario $H$. mauryi no presenta manchas en las patas y el pigmento de los segmentos caudales es tenue. La pinza de los pedipalpos del macho en $H$. mauryi es ligeramente más robusta (relación largo/ancho $=2,7$; en $H$. bustamantei $n$. sp. varía de 3,09 a 3,26). El hemispermatóforo es similar en ambas especies.

Descripción.- La coloración general es castaña-amarillenta, con manchas cafés, excepto el segmento caudal V, telson y la pinza del pedipalpo, que presentan una tonalidad castańa; parte ventral del prosoma y mesosoma, así como las patas y quelíceros castaño amarillentos; peines amarillentos. Caparax bien pigmentado, especialmente sobre las granulaciones y hacia los laterales; cúpula ocular negruzca, surco longitudinal anterior pigmentado con los bordes más oscuros. Tergitos: I-VI con cuatro manchas irregulares (dos paramedianas y dos laterales, fig. 7), en conjunto forman bandas longitudinales de pigmento; borde de los tergitos con pigmento (en algunos casos el pigmento lateral se junta ligeramente al paramediano en la parte anterior); pretergitos pigmentados, excepto la parte mediana. Tergito VII con las manchas siguiendo el patrón de los otros tergitos, pero con el pigmento menos notorio. Esternitos sin pigmento, excepto el esternito $\mathrm{V}$ que presenta dos manchas laterales alargadas sobre las carenas y cuatro pequeñas manchitas paramedianas (estas manchitas bordean la inserción de las setas). Segmentos caudales I-IV con los gránulos de las carenas oscuros; dorsalmente sin pigmento evidente, salvo ligeras manchas sobre las carenas DL en el segmento I y II de algunos ejemplares. Porción telescópica con ligeras manchas. Caras laterales con pigmento sobre los gránulos de las carenas (menos evidente sobre las LsM). Cara ventral con pigmento sobre las carenas $V_{L}$, con pequeńas manchitas que bordean la inserción de las setas en posición paramediana. Segmento V: cara dorsal con un tenue pigmento sobre las carenas DL, las manchas confluyen distalmente al pigmento lateral; caras laterales con pigmento lleno sobre las carenas $V_{L}$, éstas confluyen en la mitad distal con una banda de pigmento de posición latero-mediana; cara ventral con pigmento sobre las carenas $V_{L}$ y $V_{M}$, con manchas de pigmento en la inserción de las setas. Telson sin pigmento evidente, aguijón oscurecido. Quelíceros con pequeñas manchas cerca a la base de los dedos. Pedipalpos con la granulación oscura, fémur con un tenue pigmento cerca al borde distal (cara dorsal), patela con un ligero pigmento castaño en los bordes (cara dorsal), pinza sin pigmentación evidente. Patas con manchitas prolaterales.

Morfología.- Caparax con el tegumento granuloso, especialmente la parte lateral y posterior (tercio anterior sin granulaciones); borde anterior con una prominencia mediana, surco longitudinal anterior y posterior completos. Se puede distinguir en el caparax las carenas Mc y Lc, las MA se aprecian solamente en la parte proximal a la cúpula ocular. Tergitos I-VI finamente granulosos, siendo más evidente en el borde posterior (mayor número de gránulos en las zonas pigmentadas). Tergito VII muy granuloso, con cuatro carenas bien marcadas; los gránulos son mayores en los espacios intercarenales. Esternitos I-V lisos, en los machos con una granulación roma muy fina. Esternito $\mathrm{V}$ con dos carenas laterales (menos marcadas en las hembras). Segmentos caudales I-IV: carenas con los gránulos bien notorios; carenas DL y Lsm completas, con el gránulo distal ligeramente mayor; carena LIM completa en el segmento I, presente en la mitad distal del segmento II, en el tercio distal del segmento III, y en IV presenta sólo algunos gránulos distales; carenas $\mathrm{V}_{\mathrm{L}}$ completas en los segmentos I-IV, aunque en II-IV con pocos gránulos (fig. 1); VsM ausente en I-III, vestigial en IV; cara dorsal y espacio entre DL y LsM con algunos gránulos en I-III (especialmente en I), adicionalmente existe entre Lsm y LIM algunos gránulos distales. Segmento caudal V: carenas DL completas, VL y VM completas y con gránulos notorios (figs. $1-2,8-9)$, VsM confundida entre la granulación ventral; LM ausente. Telson con algunos gránulos ventrales proximales (figs. $1,8)$. Pedipalpos con numerosas setas: fémur con tres carenas 




Figuras 1-7.- Hadruroides bustamantei n. sp.: 1-4, holotipo macho (MHNC); 1, segmento caudales IV—V y telson, vista lateral; 2, segmento caudal V, vista ventral; 3-5, pinza derecha, 3, vista ventral, 4, vista ventromedial, 5, vista lateral; 6, dedo móvil, detalle de la dentición. 7, paratipo hembra (MHNC): pigmento del tergito V. Escalas: $1 \mathrm{~mm}$. 


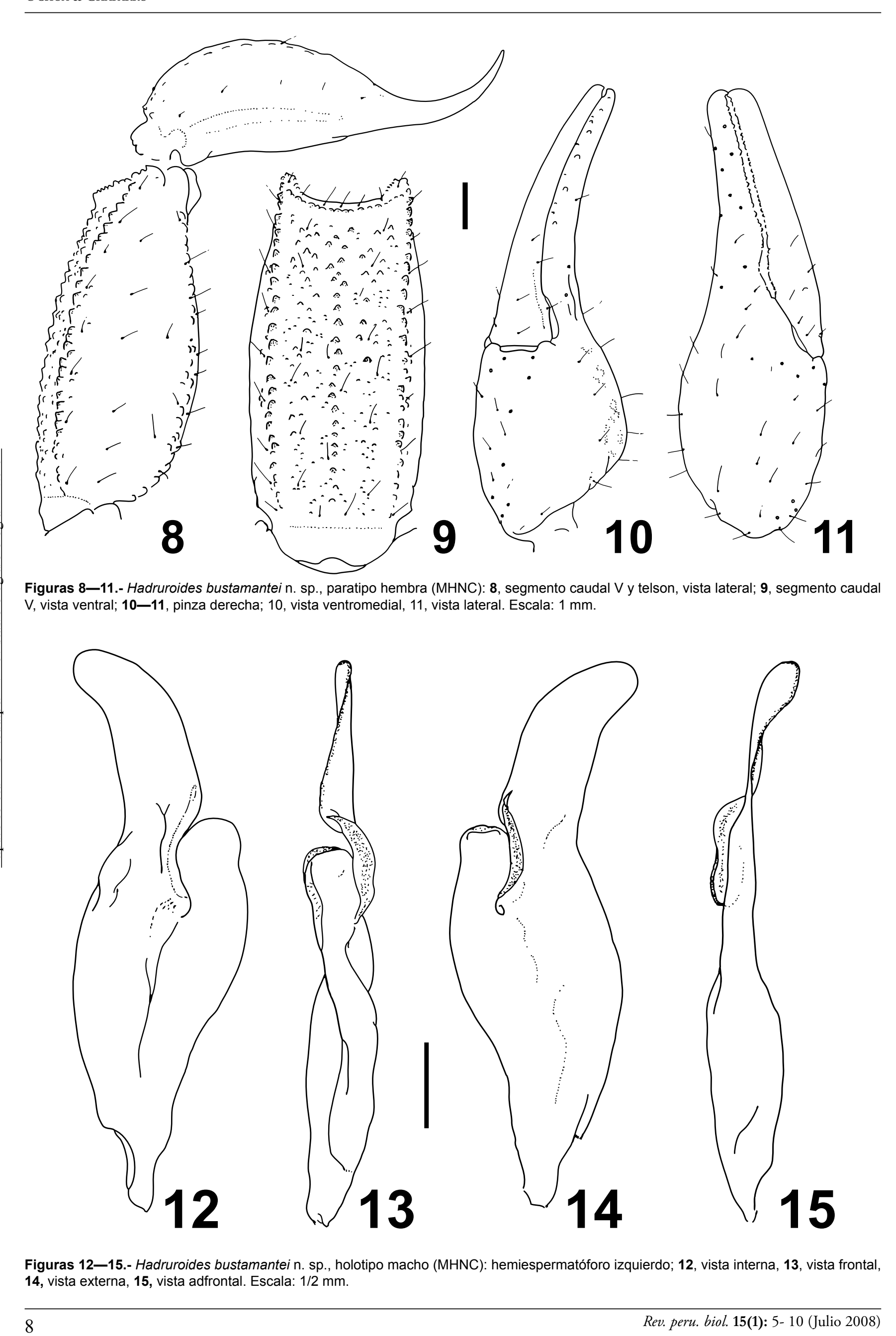




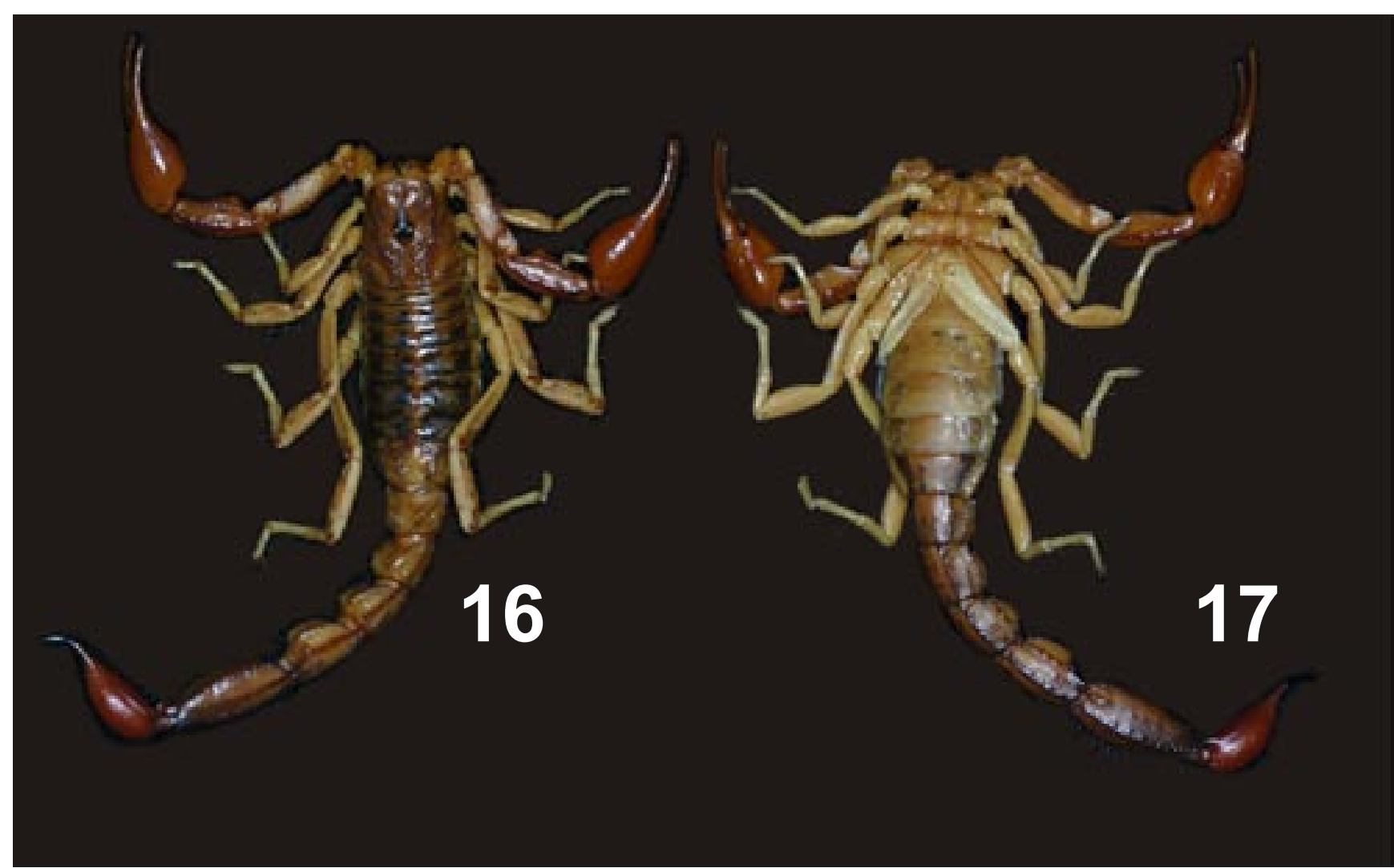

Figuras 16-17.- Hadruroides bustamantei n.sp., paratipo macho (MHNC): 16, vista dorsal, 17, vista ventral.

bien marcadas: Di, De y VI; cara dorsal e interna con algunos gránulos, cara ventral con gránulos cerca a la carena VI. Patela con las carenas Di y De marcadas; cara interna granulosa, resto de las caras lisas. Pinza redondeada y lisa, sin granulaciones; lóbulo y escotadura de la base de los dedos bien marcada en los machos, en las hembras poco desarrolladas (figs. 3-5, 10-11). Tricobotriotaxia como la del género: fémur con 3 tricobotrias $(i, e, d)$, patela con 20 tricobotrias: interna $(i)$, dorsales $\left(d_{1-2}\right)$, ventrales $\left(v_{1-3}\right)$ y 14 tricobotrias externas $\left(e t_{1-3}\right.$, est, em 1-3 $\left._{1-2} e s b_{1-2}, e b_{1-5}\right)$, pinza con 26 tricobotrias: 14 en la mano $\left(E t_{1-5}, E s t, E s b, E b_{1-3}\right.$, $\left.V_{1-4}\right)$ y 12 sobre el dedo fijo $(D t, D b, e t, e s t, e s b, e b, d t, d s t, d s b, d b$, $i t, i b)$. Número de dientes pectíneos: machos 19-21, hembras 14-19. Hemiespermatóforo lameliforme de estructura simple, lamina ligeramente curvada, porción basal ensanchada, no presentan la sutura articular ni una región capsular bien definida,

Tabla 1. Medidas (mm) de Hadruroides bustamantei n. sp. macho y hembras paratipos (MHNC)

\begin{tabular}{lcc}
\hline & macho & hembra \\
\hline Longitud total & 49,5 & 44,8 \\
Caparax, largo & 7,1 & 6,3 \\
Mesosoma, largo & 12,1 & 11,8 \\
Metasoma, largo & 30,3 & 26,7 \\
Segmento caudal I, largo & 2,3 & 2,5 \\
Segmento caudal II, largo & 2,9 & 2,9 \\
Segmento caudal III, largo & 3,4 & 3,0 \\
Segmento caudal IV, largo & 4,5 & 4,0 \\
Segmento caudal V, largo & 7,4 & 6,7 \\
Telson, largo/ancho & $9,8 / 2,9$ & $7,6 / 2,3$ \\
Pinza pedipalpo, largo/ancho/alto & $11,4 / 3,5 / 3,6$ & $9,5 / 2,72,8$ \\
\hline
\end{tabular}

en su lugar existe una escotadura bien pronunciada ubicada aproximadamente en la parte media, acompańada externamente de una cresta alargada; adicionalmente existe un lóbulo libre que presenta un ligero repliegue hacia el lado frontal (figs. 12-15).

\section{Variabilidad}

- Longitud total: machos 44,0—49,5 mm; hembras hasta 47,6 mm. (Tabla 1)

- Frecuencia del número de dientes pectíneos: machos ( $\mathrm{n}=10$ péctenes): 19 dientes (4 péctenes), 20 (5), 21 (1); hembras $(\mathrm{n}=20), 14$ (1), 15 (1), 16 (5), 17 (11), 18 (1), 19 (1).

- Alto relativo del telson: índice largo / alto: machos, 3,07-3,38 $($ media $=3,25 ; n=5)$; hembras, 3,16-3,47 (media=3,31; $\mathrm{n}=7$ ).

- Índice largo / ancho de la pinza: machos, 3,09-3,26 (media $=3,17 ; n=5)$; hembras, 3,17-3,57 (media=3,40; $n=7)$.

- Índice largo / alto de la pinza: machos, 2,96-3,14 (media=3,034; $n=5)$; hembras, 3,04-3,41 (media=3,24; $n=7)$.

Distribución y hábitat.- Valles interandinos en los Andes centrales de Perú en los departamentos de Ayacucho y Huancavelica entre 2600 a 3289 m (fig. 18). Esta especie vive en terrenos pedregosos con poca vegetación arbustiva de tipo xerofítica, en todos los casos los ejemplares fueron encontrados debajo de piedras. Al igual que $H$. mauryi, la nueva especie se distribuyen también en valles interandinos, sin embargo, $H$. bustamantei n. sp. está presente en ambientes xerofíticos correspondiente a los "valles interandinos cálidos", mientras que $H$. mauryi se distribuye en ambientes de "queswa" (Ochoa, 2005).

Comentario.- Originalmente $H$. mauryi fue descrito en base a un único ejemplar macho adulto (holotipo) y un juvenil (paratipo) proveniente de la localidad de Paruro en el departamento del Cusco, además de varias hembras y juveniles (paratipos) de 


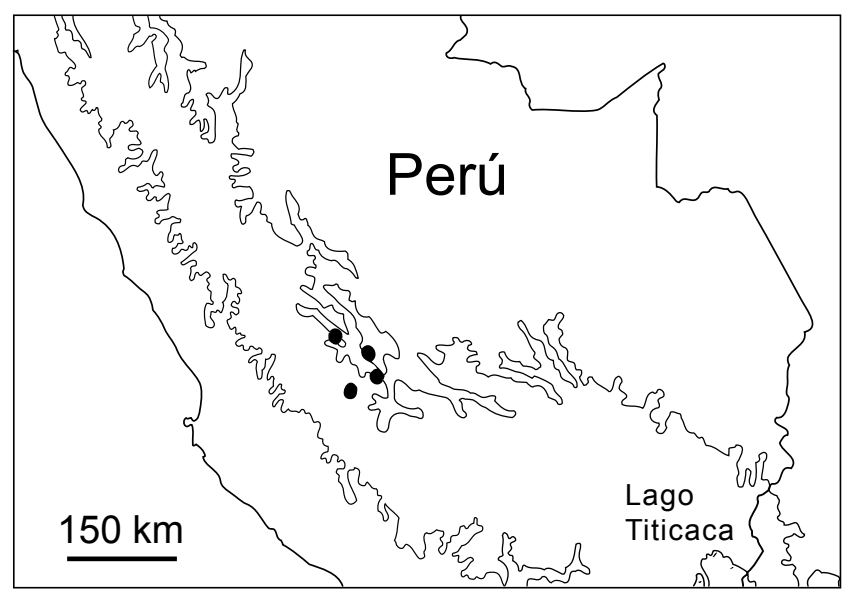

Figura 18.- Distribución geográfica de Hadruroides bustamantei n.sp. en el Perú. Cota de altitud 3000 m aproximadamente.

los departamentos de Huancavelica y Ayacucho; sin embargo el material de estos dos últimos departamentos corresponden a $H$. bustamantei n.sp. Francke \& Soleglad (1980) confundieron ambas especies probablemente por la ausencia de ejemplares machos de la zona de Ayacucho y la no disponibilidad de hembras adultas de la localidad tipo. Ningún autor posterior revisó el material tipo de H. mauryi (Sissom \& Fet, 2000; Ochoa, 2003) y por tanto siguieron el mismo criterio de Francke \& Soleglad.

Etimología.- Esta especie está dedicada al Dr. Javier Bustamante, en reconocimiento a su apoyo a la investigación taxonómica y conservación de la biodiversidad en el Perú.

\section{Otros materiales estudiados:}

H. bustamantei n. sp. (no tipos): Perú, departamento Ayacucho: 1 hembra (MUSM), Huanta (2600 m), abril 1949, F. Blancas; 1 macho, 1 hembra (MHNC), Ruinas de Huari, febrero 2005, E. Escobar; 1 hembra (MHNC), Vinchos (3289 m), 01 diciembre 2006, W. Mendoza \& M. Medina; 1 hembra, 1 juvenil (MUSM), casa Urcco, ca. Ayacucho (3000 m), 12 febrero 1963, R. Garcia.

H. mauryi: Perú, departamento Cusco: holotipo macho (AMNH), 1 juvenil paratipo (AMNH), Paruro, Departamento Cusco, Perú; 28 octubre 1966; A. Guerra.

\section{Agradecimientos}

Agradecemos a Javier Bustamante y su familia por el apoyo y soporte a esta investigación. Agradecemos también a Lorenzo Prendini (AMNH) por el préstamo del material tipo de $H$. mauryi y a Gerardo Lamas por facilitar la colección del MUSM. A John Achicahuala por la ayuda en tareas de colecta. Enrique Escobar, Wilfredo Mendoza y Margarita Medina, facilitaron material adicional proveniente de Ayacucho. Asimismo agradecemos a IDEA WILD por proporcionarnos un estereomicroscopio para el desarrollo de este trabajo.

\section{Literatura citada}

Acosta, L. E. \& J. A. Ochoa. 2002. Lista de los escorpiones bolivianos (Chelicerata: Scorpiones), con notas sobre su distribución. Revista de la Sociedad Argentina de Entomología. $61(3-4): 15-23$.
Aguilar, P. G. \& O. Meneses. 1970. Escorpiones y Escorpionismo en el Perú I: Nota preliminar sobre los Scorpionida peruanos. Anales Científicos de la Universidad Nacional Agraria La Molina, 8: 1-5.

Fet, V. \& M.E. Soleglad. 2005. Contributions to Scorpion Systematics. I. On Recent Changes in High-Level Taxonomy. Euscorpius 31: 1-13.

Flórez, E. 1990. Escorpiones de Colombia. Catálogo de especies. Cespedesia, 16-17 (57-58): 117-127.

Francke, O. F. \& M. E. Soleglad. 1980. Two new Hadruroides Pocock, from Peru (Scorpiones-Vaejovidae). Occasional Papers of the Museum, Texas Tech University, 69: 1-13.

Francke, O. F. \& M. E. Soleglad. 1981. The family Iuridae Thorell (Arachnida, Scorpiones). Journal of Arachnology, 9: 233 $-258$

Karsch, F. 1879. Scorpionologische Beiträge. Part II. Mitteilunger des Münchener Entomologischen Vereins, 3: 97-136.

Kraepelin, K. 1894. Revision der Scorpione. II. Scorpionidae und Bothriuridae. Beiheft zum Jahrbuch der Hamburgischen Wissenschaftlichen Anstalten, 11: 1-248.

Kraepelin, K. 1899. Scorpiones und Pedipalpi. In F. Dahl (ed.), Das Tierreich. Herausgegeben von der Deutschen Zoologischen Gesellschaft. Berlin: R. Friedländer und Sohn Verlag, Vol. 8: 1-265.

Lourenço, W. R. 1995. Les scorpions (Chelicerata, Scorpiones) de l'Équateur avec quelques considérations sur la biogéographie et la diversité des espèces. Revue Suisse de Zoologie, 102 (1): 61-88

Maury, E. A. 1975. Escorpiones y escorpionismo en el Perú IV: Revisión del género Hadruroides Pocock, 1893 (Scorpiones, Vejovidae). Revista Peruana de Entomología, 17 (1): 9-21.

Mello-Leitão, C. 1945. Escorpiões sul-americanos. Arquivos do Museu Nacional, 40: 7-468.

Ochoa, J. A. 2003. Sistemática y patrones de distribución de los escorpiones (Chelicerata) del corredor andino, en el sur del Perú. Tesis doctoral. Facultad de Ciencias Exactas, Físicas y Naturales, Universidad Nacional de Córdoba, i-iv, $259 \mathrm{pp}$

Ochoa, J.A. 2005. Patrones de distribución de escorpiones de la región andina en el sur peruano. Revista Peruana de Biología, 12 (1): 49-68.

Prendini, L. \& W. C. Wheeler. 2005. Scorpion higher phylogeny and classification, taxonomic an-archy, and standards for peer review in online pub-lishing. Cladistics, 21: 446-494.

Sissom, W. D. \& V. Fet. 2000. Family Iuridae Thorell, 1876. In: Fet, V., W. D. Sissom, G. Lowe \& M. E. Braunwalder. Catalog of the Scorpions of the World (1758-1998). Pp. 409-420. The New York Entomological Society, New York.

Soleglad, M. E. \& V. Fet. 2003. High-Level Systematics and Phylogeny of the Extant Scorpions (Scorpiones: Orthosterni). Euscorpius, Occasional Publications in Scopiology, 11: 175.

Stahnke, H. L. 1970. Scorpion nomenclature and mensuration. Entomological News, 81: 297-316.

Vachon, M. 1952. Étude sur les Scorpions. Institut Pasteur d'Algérie, Alger, $482 \mathrm{pp}$.

Vachon, M. 1974. Étude des caractères utilisés pour classer les familles et les genres de scorpions (Arachnides). 1. La trichobothriotaxie en Arachnologie. Sigles trichobothriaux et types de trichobothriotaxie chez les Scorpions. Bulletin du Muséum National d'Histoire Naturelle, Paris, (3), 140 Zool. 104: 857-958. 